Archives

$43 \mid 2009$

Hommage à Jacques Ozouf

\title{
Travailler avec Jacques Ozouf
}

\section{Pierre Nora}

\section{OpenEdition \\ Journals}

Édition électronique

URL : http://journals.openedition.org/ccrh/3499

DOl : $10.4000 /$ ccrh.3499

ISSN : 1760-7906

Éditeur

Centre de recherches historiques - EHESS

Édition imprimée

Date de publication : 25 janvier 2009

Pagination : 57-62

ISSN : 0990-9141

Référence électronique

Pierre Nora, «Travailler avec Jacques Ozouf », Les Cahiers du Centre de Recherches Historiques [En ligne], 43 | 2009, mis en ligne le 16 novembre 2011, consulté le 23 avril 2019. URL : http:// journals.openedition.org/ccrh/3499; DOI : 10.4000/ccrh.3499

Ce document a été généré automatiquement le 23 avril 2019.

Article L.111-1 du Code de la propriété intellectuelle. 


\title{
Travailler avec Jacques Ozouf
}

\author{
Pierre Nora
}

1 En écoutant les précédentes interventions, j'ai pris davantage encore conscience de la proximité et même de l'intimité que j'ai eu la chance d'entretenir avec le travail de Jacques Ozouf : comme ami, comme historien et comme éditeur.

2 Nous les Maîtres d'école, pour commencer, a paru dans cette petite collection de poche « Archives » que j'ai créée en 1964 et qui était destinée, d'une manière neuve à l'époque, à présenter des pièces dites d'archives, des documents directs montés par les meilleurs des historiens. Il y avait déjà eu ainsi Les Français ont la parole, présentation des Cahiers des États Généraux de Pierre Goubert et l'An Mil de Georges Duby, Auschwitz de Léon Poliakoff ou le Congrès de Tours par Annie Kriegel. Je savais, par l'amitié quotidienne avec Mona et Furet, le travail extraordinaire qu'était en train de récolter Jacques. Dans cette petite collection on avait jusqu'à présent eu affaire qu'à des documents déjà établis, qu'il s'agissait de rassembler. Là, il y avait de toute évidence un trésor constitué, d'une richesse inattendue, puisque sur 20.000 lettres envoyées, 4.000 avaient été honorées d'une réponse qui dépassait de beaucoup le questionnaire. Il s'agissait d'un milieu spécial d'hommes et de femmes qui avaient appris à écrire eux-mêmes et aux autres et qui dans l'esprit à la fois de vérité, de nostalgie, d'autobiographie avaient le sentiment qu'il y avait un continent dont ils étaient les sauveurs grâce à la bouée que leur lançait un plus jeune. Et quel plus jeune! Il portait le nom d'un des leurs, Ozouf. Je suis persuadé que Jacques n'aurait pas eu cette moisson s'il n'avait porté ce nom de haute généalogie scolaire. Ces retraités lui avaient donc envoyé des journaux qu'ils tenaient à l'époque indépendamment $\mathrm{du}$ questionnaire; et il s'était trouvé devant une masse presque encombrante qu'il ne savait pas encore très bien comment mettre en œuvre. C'était l'époque des thèses monumentales et interminables. En attendant, il était fait pour « Archives ». Je le pressais de passer à l'acte. Peut-être l'aurait-il fait plus tôt s'il n'était surveillé par l'œil amicalement réprobateur de François Furet qui devait trouver qu'il ferait mieux de continuer à travailler sérieusement plutôt que de s'intéresser à cette collection sur laquelle il portait un jugement ironique. Oserai-je le dire ici ? Considérant qu'il s'agissait d'un travail très facile de rassemblement de documents et me voyant me 
jeter dans tous les sens pour avoir des auteurs, François Furet appelait aimablement cette collection " le râteau à cons »; si bien que Jacques Ozouf m'a dédicacé son volume avec cette charmante formule : « Une dent du râteau »!

3 Eh bien, ça été l'ouvrage le plus neuf et peut être le meilleur de la collection, en 1967. Il a eu un succès à peu près analogue à celui de Auschwitz, ce qui voulait dire que dans la conscience collective et à des titres très différents, le thème même de l'instituteur était en train de trouver des échos profonds. Il ne faut pas oublier que c'était l'époque où ce thème avait une légère connotation politique. Nous sommes en période gaulliste et l'instituteur est une figure éponyme de la République combative et conquérante. C'était l'époque où je venais de faire un essai sur Lavisse et le sentiment national, où Mona Ozouf travaillait sur le patriotisme dans les manuels scolaires, où Aghulon réhabilitait l'étude de la République, où Georges Duveau nous avait appris le respect de ces instituteurs. Autour de cette figure se dessinait une constellation que l'on va retrouver dans un instant avec le journal de Vincent Auriol dont les connotations anti-Cinquième République étaient évidentes.

4 Voilà dans quelles conditions un peu particulières le livre sur les instituteurs de la Belle Époque avait paru sans que nous nous posions trop de problèmes de méthodologie, de sciences sociales, de mémoire et d'autobiographie. Mais avec le sentiment d'un contact direct avec le passé à travers les documents, conscience de la difficulté d'un montage subtil et parlant, et volonté de faire partager au grand public du livre de poche ce contact rafraîchissant avec le passé.

5 Il y a un autre travail de Jacques auquel je me sens très lié, c'est son article sur l'opinion et les sondages dans Faire de l'histoire en 1973. C'était une réponse à Pierre Bourdieu. La culture politique française avait été, on le sait, longtemps réfractaire à la technique des sondages. Elle a explosé à un moment très particulier : en 1965, l'année de la première élection au suffrage universel du président de la République. C'est donc dans les années suivantes que le problème a été ardemment discuté et vigoureusement dénoncé par Bourdieu dans Les Temps Modernes. L'article de Ozouf est une apologie et une défense argumentée, un petit traité du bon usage des sondages pour l'étude de l'opinion publique dont il montre que l'on ne peut parler au singulier. Nous l'avons placée, Jacques Le Goff et moi, dans le volume consacré aux « Nouveaux objets ».

6 C'est surtout de l'aventure du Journal du septennat de Vincent Auriol que je m'étais proposé de parler. Là encore j'y suis moi-même tellement lié que je me vois obligé de vous en rappeler les circonstances. Je crois que c'est en 1967, au moment où Jacques a publié Nous les Maîtres d'école, que la veuve du président de la République s'est adressée à moi par personne interposée. Elle m'a raconté que son mari, dans son intention d'écrire ses mémoires, durant son septennat de 1947 à 1953, avait pris des notes après chacune des visites de ses interlocuteurs, espaçant ses rendez vous de quelques minutes. En 1950 quelqu'un lui avait apporté des États-Unis à titre de gadget un petit magnétophone. Pour garder la fidélité des conversations et pour gagner du temps, il avait installé l'appareil dans son bureau et le déclenchait dès que son interlocuteur prenait la parole. Sa secrétaire arrivait deux heures avant lui le matin pour dactylographier l'enregistrement. Au bout de sept ans, le Président s'était trouvé devant une masse considérable d'archives qu'il n'avait pas su ou pas pu maîtriser avant de disparaitre. La pièce dans laquelle elle m'avait introduite m'a rappelé immédiatement celle dans laquelle Jacques Ozouf entassait les dossiers de ses instituteurs. Même ampleur, même désordre. En travaillant là quelques jours, j'ai découvert un monde extraordinaire. Par rapport au secret qui pesait sur toutes 
les archives d'histoire contemporaine, il y avait là une manne, une mine d'une originalité absolue. Dans une scène politique où les acteurs ne cessaient de se relayer rapidement, Vincent Auriol représentait la seule permanence. Avec lui, on vivait la Quatrième république de l'intérieur ! Il y avait là tous les procès-verbaux des conseils de ministres, du Haut conseil de la magistrature, du conseil de l'Union française, de la DGRST, les notes des Affaires étrangères secrètes, un dossier de police quotidien qui arrivait sur le bureau du Président à l'époque où les communistes étaient au pouvoir entre 1946 et 1947. De quoi tourner la tête d'un historien de la France contemporaine. Au bout de huit jours Madame Auriol m'a demandé mon verdict et je lui ai dit que c'était tellement intéressant que ça me paraissait mériter une publication intégrale et critique. Elle a eu cette réaction charmante : «Mais Monsieur, mon mari était au-dessus de toute critique !» Cela dit, elle m'a laissé carte blanche et constamment fait confiance.

7 C'est là qu'arrive Jacques. Devant cette énorme masse, 15 à 20.000 pages, souvent illisibles, en désordre, les premières années manuscrites, un très gros travail s'imposait. Je me suis tourné tout naturellement vers lui pour lui proposer de s'associer à moi, d'abord parce qu'il était bien plus connaisseur que je ne l'étais moi-même. Il était, lui, l'homme désigné pour mener à bien cette immense entreprise.

Nous avons tout de suite décidé qu'il fallait consacrer un volume par année du septennat, me chargeant, moi, de la première, 1947, qui établirait les normes de l'édition, Jacques Ozouf se chargeant de la dernière année, 1952-1953 qui permettait ainsi de baliser l'ensemble. Il était entendu qu'il trouverait des collaborateurs pour les années 1950 et 1951, moi pour les années 1948 et 1949.

9 Indépendamment de son intérêt historique évident, l'entreprise avait un caractère d'actualité politique, et même légèrement polémique. Elle apparaissait comme une discrète réhabilitation de la Quatrième république par rapport à la Cinquième, mais surtout comme une apologie de la République elle-même, à travers son premier président socialiste. Vincent Auriol s'y révélait en effet, dans cette lutte au jour le jour, pleine d'espoir au lendemain de la Libération et de plus en plus amère, comme un homme très différent de ce que son bon sourire, son accent savamment entretenu, son naturel sympathique et bon enfant avaient construit comme image de lui. Il apparaissait au contraire comme beaucoup plus énergique, construisant obstinément une magistrature d'influence unique en son genre, et portant sur les institutions qu'il avait largement contribué à établir et les hommes du milieu politique, un regard sévère et critique. Mais c'était surtout le caractère inattendu de ce Journal au jour le jour, pas même composé par lui, qui en faisait l'antithèse des mémoires du Général de Gaulle, que l'on attendait incessamment. L'opposition entre les deux hommes en devenait plus parlante. Je savais que Jacques se passionnerait pour l'entreprise. J'ai pu vérifier tous les jours sa connaissance intime de la vie politique, sa familiarité avec le personnel politique, son érudition ironique et infaillible, sa mémoire des dates et des détails, son sens des situations et du rapport des forces. Il faisait aussi l'admiration de René Rémond, qui n'a cessé de s'intéresser de près à notre travail et qui a d'ailleurs écrit la présentation de l'ensemble.

10 Comme l'édition intégrale, engagée chez Armand Colin, allait prendre des années et qu'il était dommage de la réserver aux seuls spécialistes, il nous a paru indispensable d'en faire une édition publique et réduite qui sortirait en même temps que mon année 1947. C'est là qu'il faut insister sur le travail et le talent de Jacques Ozouf. Le volume Mon septennat est signé de nos deux noms, mais comme j'étais très occupé par l'année 1947, l'essentiel de la 
paternité du volume chez Gallimard, dans la collection « Témoins », revient à Jacques : il y fallait une maîtrise de l'ensemble du corpus pour y choisir les extraits les plus significatifs et assurer les présentations pour lier l'ensemble en un tout cohérent.

11 La sortie simultanée des deux volumes, qui ont connu un grand succès, a manqué ne pas se faire.

12 Pendant que je travaillais en effet, j'avais eu l'occasion de dîner avec Georges Pompidou, juste avant sa fameuse "déclaration de Rome", où il se déclarait éventuellement candidat à la succession du Général de Gaulle. Quand je lui avais dit, sur sa demande, ce que j'étais en train de faire, il s'était emporté : « Mais c'est un scandale !»; et si l'on pense aux réactions, ou plutôt au manque de réactions politiques et présidentielles à la parution des Verbatim de Jacques Attali, on mesure le chemin parcouru. «Je comprends votre bonheur d'historien, c'est très intéressant. Mais je suis scandalisé que Madame Auriol ait pu vous laisser faire une chose pareille ». C'est ce qui nous a fait nous entourer, Jacques et moi, d'un comité de patronage de haute qualité : Pierre Renouvin, Julien Cain, François Goguel, Georges Vedel et consulter évidemment un avocat, notre ami Georges Kiejman; bref nous avancer entourés de toutes les précautions scientifiques et juridiques.

Elles n'ont pas empêché les estafettes de l'Elysée de venir chercher les épreuves; car entre temps, Georges Pompidou était devenu président de la République. Nous avons vécu dans l'incertitude pour apprendre quelques jours après que Georges Pompidou avait eu la ferme intention de faire saisir. Papiers d'Etat, procès-verbaux de conseils de ministres, secrets de défense nationale, c'était la première fois qu'ils étaient publiés sous cette forme, au grand jour. L'affaire devenait «nationale». Une consultation avait été demandée d'urgence au Conseil d'État, qui concluait à la saisie. Et c'est, parait-il, à Pierre Juillet et à Marie-France Garaud que nous avons dû notre salut. Ils avaient conseillé la prudence au prétexte que les Mémoires du Général de Gaulle, qui devaient paraître quelques mois plus tard, risquaient de comprendre aussi, en annexe, des documents d'État, et que si la Présidence de la République faisait saisir Auriol, cela aurait eu l'air que la Cinquième république intente un procès à la Quatrième. À contrecœur, Georges Pompidou avait finalement laissé faire.

14 Et c'est ainsi que Jacques et moi avons pu tranquillement faire sortir l'année 1948 par Jean-Pierre Azéma, 1949 par Pierre Kerleroux, tandis que Dominique Boché se chargeait de l'année 1952, Laurent Theis de l'année 1951. Anne-Marie Bellec allait publier l'année 1950 pour clore l'entreprise, quand l'affaire a connu un étrange rebondissement ou plutôt, un étrange étouffement.

15 Cette fois, c'était François Mitterrand qui était entre temps devenu président de la République. Il avait eu la curiosité de lire ce que Vincent Auriol avait dit de lui quand il était ministre de la Quatrième république, et ses appréciations peu amènes ne lui avaient pas plu. Du coup, lorsque avait été demandé par Armand Colin le soutien du CNL, qui nous était régulièrement accordé, nous nous sommes heurtés à un refus obstiné. Aucune démarche n'a abouti, ni l'intervention de Pierre Bérégovoy, ni la visite de Paul Auriol, le fils de Vincent, à François Mitterrand. Il a donc fallu attendre la fin des deux septennats pour reprendre le problème. Mais au fil du temps, bien des choses avaient changé.

16 Armand Colin avait changé de ligne éditoriale, la Quatrième république n'avait plus la même actualité politique, les six autres volumes étaient épuisés, le manuscrit d'AnneMarie Bellec exigeait une mise à jour. Et Jacques, qui ne pouvait plus s'intéresser à cette publication que de loin, n'en a pas vu, hélas l'épilogue heureux. 
17 J'avais raconté cette histoire dans une conférence aux Archives nationales à laquelle assistait Henri Bovet, le nouveau directeur des éditions Tallandier. Il m'a fait une proposition qui a abouti, il y a deux ans, à la meilleure des solutions : la publication de l'année 1950 accompagnée d'un CD comportant la réédition des six volumes. Je regrette infiniment que Jacques n'ait pas été de cette ultime aventure à laquelle son nom restera attaché.

Je me suis souvent demandé ce qu'aurait fait Jacques s'il avait pu poursuivre son œuvre. Il appartenait à cette poignée de très rares historiens journalistes qui savent intervenir sur l'actualité avec le regard et la culture de l'historien. Ils ne sont pas nombreux. Il y avait Furet, qui n'est plus là. Il y a Jacques Julliard, qui est heureusement bien là. Ozouf se distinguait par un savoir-faire sensible et savant, une analyse toute en finesse des forces et de la vie politiques. J'en ai, tout récemment, retrouvé une preuve. Republiant dans la collection « Témoins » le témoignage d'Emmanuel Berl sur le 10 juillet 1940 initialement paru dans «Les Trente journées qui ont fait la France » en mai 1968 - drôle de date ! - je cherchais à l'accompagner d'annexes et des critiques qui l'avaient accueilli. Le long compte rendu que Jacques en a publié en octobre dans l'Observateur, où Berl n'avait jusque-là pas droit de séjour pour avoir écrit le premier discours de Pétain, est un chefd'œuvre de sensibilité, de distance critique, d'empathie intellectuelle. Je ne crois pas que les deux hommes se soient jamais rencontrés et je regrette de ne pas les avoir rapprochés, car ils se seraient merveilleusement entendus dans le commentaire perpétuel de l'histoire et de l'actualité politique. Je vous renvoie à cet article, Jacques y est tout entier. À propos, ne serait ce pas une bonne idée de réunir, comme Mona l'a fait pour Furet, les articles, chroniques et comptes rendus de Jacques? Pardonnez à l'historien de finir une fois de plus en éditeur.

\section{AUTEUR}

PIERRE NORA

EHESS/CRH 